\title{
Application of Bounded Linear Stability Analysis Method for Metrics-Driven Adaptive Control
}

\author{
Maryam Bakhtiari-Nejad* \\ Nhan T. Nguyen ${ }^{\dagger}$ \\ Kalmanje Krishnakumar \\ NASA Ames Research Center, Moffett Field, CA 94035
}

\begin{abstract}
This paper presents the application of Bounded Linear Stability Analysis (BLSA) method for metrics-driven adaptive control. The bounded linear stability analysis method is used for analyzing stability of adaptive control models, without linearizing the adaptive laws. Metrics-driven adaptive control introduces a notion that adaptation should be driven by some stability metrics to achieve robustness. By the application of bounded linear stability analysis method the adaptive gain is adjusted during the adaptation in order to meet certain phase margin requirements. Analysis of metrics-driven adaptive control is evaluated for a $2^{\text {nd }}$ order system that represents a pitch attitude control of a generic transport aircraft. The analysis shows that the system with the metrics-conforming variable adaptive gain becomes more robust to unmodeled dynamics or time delay. The effect of analysis time-window for BLSA is also evaluated in order to meet the stability margin criteria.
\end{abstract}

\section{Introduction}

Adaptive control laws are generally nonlinear and therefore stability robustness of adaptive control cannot be analyzed by linear stability metrics in terms of phase and gain margins. These margins are designed into linear control laws to provide robustness to account for system uncertainties such as modeling errors and exogenous disturbances. The lack of stability metrics for adaptive control is a major challenge to enable adaptive control laws to be adopted in production control systems. Metrics-driven adaptive control introduces a notion that adaptation should be driven by some stability metrics to achieve robustness. ${ }^{1}$ The bounded linear stability analysis method is applied in order to analyze adaptive control in terms of the linear stability concept by establishing an approximate linear equivalent system as a function of a mean square value of teh input fucntion to the adaptive law. ${ }^{2}$ The method uses an error bound analysis to extract the dominant linear components of the nonlinear adaptive laws without linearization. The idea is to seek a linear representation that bounds a nonlinear adaptive law. This method can provide an understanding of the stability margin of nonlinear adaptive control that can be used to establish limits on adaptive gains during adaptation to ensure system robustness, and thus the adaptation is made to be metrics-driven.

\section{Adaptive Control of a Second-Order System}

Figure 1 shows the model-reference adaptive control (MRAC) architecture for a pitch attitude control of a generic transport model (GTM) of a $2^{\text {nd }}$ order system of aircraft. The plant model is given as

$$
\dot{x}=A x+B u
$$

where $x=\left[\begin{array}{c}\theta \\ \dot{\theta}\end{array}\right]$ is the state vector, $\theta$ and $\dot{\theta}$ are pitch angle and pitch rate respectively, $A=\left[\begin{array}{cc}0 & 1 \\ -M_{q \dot{\alpha}} & M_{\alpha}\end{array}\right]$

\footnotetext{
*Aerospace Engineer, Intelligent Systems Division, Mail Stop 269-1, AIAA Member

${ }^{\dagger}$ Research Scientist, Intelligent Systems Division, Mail Stop 269-1, AIAA Senior Member

${ }^{\ddagger}$ Research Scientist, Intelligent Systems Division, Mail Stop 269-1, AIAA Associate Fellow
} 
is assumed to be unknown, and $B=\left[\begin{array}{c}0 \\ M_{\delta_{e}}\end{array}\right]$ is known.

According to R.C. Nelson, ${ }^{3} M_{q \dot{\alpha}}=M_{q}+M_{\dot{\alpha}}=\left(C_{m_{q}}+C_{m_{\dot{\alpha}}}\right) \frac{\bar{c}}{2 u_{0}} \frac{Q S \bar{c}}{I_{y}}$, and $M_{\delta_{e}}=C_{\delta_{e}} \frac{Q S \bar{c}}{I_{y}}$, where $C_{m_{q}}$, $C_{m_{\dot{\alpha}}}$, and $C_{\delta_{e}}$ are the pitching moment derivatives, $\bar{c}$ is the mean aerodynamic chord, $S$ is the wing area, $u_{0}$ is the aircraft velocity along the $x$ body axis, , and $I_{y}$ is the mass moment, and $Q=\frac{1}{2} \rho u_{0}^{2}$, where $\rho$ is the air density.

The output of the dynamic inversion $u$ is defined as

$$
u=\left(b^{T} B_{n}\right)^{-1}\left(b^{T} \dot{x}_{m}-b^{T} A_{n} x-u_{a d}+u_{p d}\right)
$$

where $b=\left[\begin{array}{l}0 \\ 1\end{array}\right], B_{n}=\left[\begin{array}{c}0 \\ M_{\delta_{e_{n}}}\end{array}\right]$ and $A_{n}=\left[\begin{array}{cc}0 & 0 \\ -M_{q_{n} \dot{\alpha}_{n}} & -M_{\alpha_{n}}\end{array}\right]$ contain the nominal parameters for the dynamic inversion, $\dot{x}_{m}$ is a model-reference acceleration, $u_{a d}$ is the adaptive control signal, and $u_{p d}$ is a proportional-derivative (PD) controller.

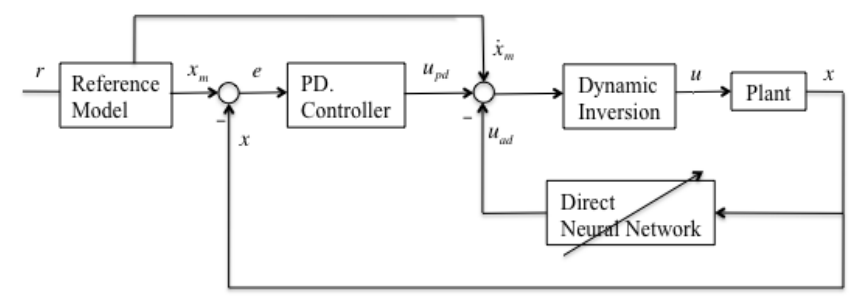

Fig.1 MRAC Architecture for Pitch Model of Aircraft.

The PD controller $u_{p d}$ is defined as

$$
u_{p d}=k e
$$

where $k=\left[\begin{array}{ll}k_{p} & k_{d}\end{array}\right]$ is a gain vector with $k_{p}$ and $k_{d}$ being the proportional and derivative gains respectively, and $e=x_{m}-x$ is the tracking error.

The adaptive signal $U_{a d}$ is parametrized by a linear-in-parameters matched uncertainty

$$
u_{a d}=W^{\top} x
$$

where $W$ is a weight matrix and $x$ is the basis function, which in the case of this example is the same as the state vector.

The adaptive law given below can be shown to be stable based on the Lyapunov stability proof. ${ }^{2}$

$$
\dot{W}=-\Gamma x e^{\top} P b
$$

where $\Gamma>0$ is an adaptive gain, and $P$ solves the Lyapunov equation as follows

$$
P A_{c}+A_{c}^{T} P=-Q
$$

where $A_{c}=\left[\begin{array}{cc}0 & 1 \\ -k_{p} & -k_{d}\end{array}\right]$ is Hurwitz, and $Q=I>0$ is a symmetric positive-definite matrix.

Figure 2 shows the tracking performance of the adaptive control system. In order to simulate an uncertainty, a 30\% reduction in the pitch damping is imposed. A constant adaptive gain of $\Gamma=100$ is used. Figure 2 shows that the tracking performance of the adaptive system with the parameteric failure is perfectly acheived. 


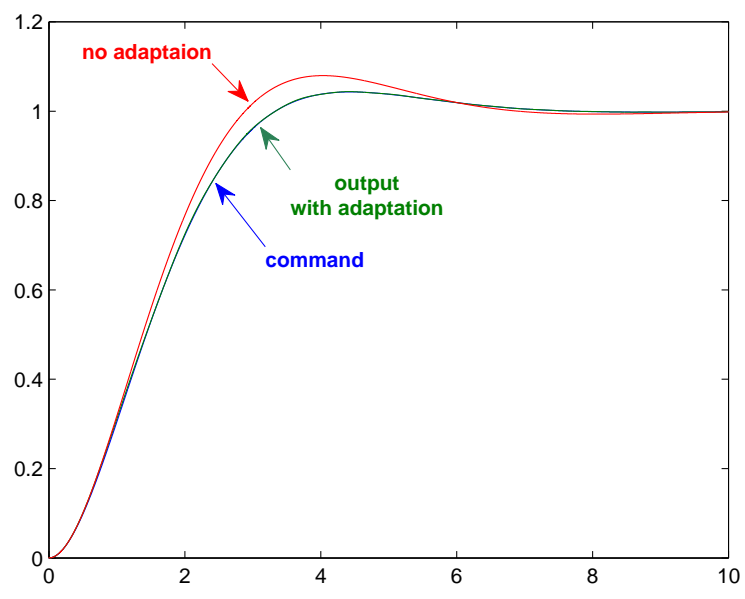

Fig.2 Tracking Performance with Reduced Pitch Damping.

\section{Bounded Linear Stability Analysis (BLSA)}

Stability of nonlinear adaptive control is usually analyzed by the Lyapunov method. The traditional linear stability margin, phase margin and gain margin concept, may be extended to nonlinear adaptive control if it could be represented by some linear approximations. The bounded linear stability analysis seeks a piecewise linear equivalent approximation of nonlinear adaptive control in terms of a mean square value of the input function to the adaptive law over a short time window during which the linear time invariant (LTI) concept of stability margins could be analyzed to provide a method for adjusting the adaptive gain for the next time window.

The stability of the adaptive law can be analyzed by a conservatively bounded linear stability using a linear equivalent adaptive law ${ }^{2}$ as follows.

Let $W^{*}$ be the constant ideal weight and $\tilde{W}=W-W^{*}$ be the weight variation, therefore

$$
\frac{d}{d t}\left(\tilde{W}^{\top} x\right) \leq-\Gamma \alpha_{0} b^{\top} P e+\Delta_{1}
$$

where $\Delta_{1}>0$ is a constant that represents a bound, and $\alpha_{0}>0$ is a mean square value of the input function to the adaptive law such that

$$
\alpha_{0} \leq \frac{1}{T} \int_{t}^{t+T} x^{\top} x d \tau \approx \frac{1}{n} \sum_{k=0}^{n-1} x^{T} x(t+k T)
$$

where $T=n \Delta t$ is the analysis time window and $n$ is the number of time steps $\Delta t$.

Since $u_{a d}=\left(W^{*}+\tilde{W}\right)^{T} x$, then the linear bound to the adaptive law (Eq.5) is rewritten as

$$
\frac{d}{d t}\left(u_{a d}\right)=-\Gamma \alpha_{0} b^{\top} P e+\Delta_{1}
$$

or

$$
u_{a d}(s)=-\Gamma \alpha_{0} \frac{P_{22} s+P_{12}}{s} \Theta_{e}(s)+\Delta_{2}
$$

where in the Laplas transform $\Theta_{e}(s)=\Theta_{c}(s)-\Theta(s)$ is the error, $\Theta_{c}(s)$ is the command, and $\Delta_{2}$ is a constant bound .

Using the bounded linear stability analysis approach the open-loop transfer function between the output $\Theta(s)$ and the error $\Theta_{e}(s)$ could easily be derived as shown below. In deriving the open-loop transfer function, the parametric uncertainty in the pitch damping $\xi$ is included. 
Substituting Eq. $\quad$ into Eq.2 and then into Eq.1 and taking the Laplace transform results in

$$
\begin{aligned}
& s X=\left(A-A_{n}\right) X+\underbrace{\Gamma \alpha_{0}\left[\begin{array}{c}
0 \\
1
\end{array}\right] \times \frac{P_{22} s+P_{12}}{s} \Theta_{e}(s)}_{u_{a d}}+\underbrace{\left[\begin{array}{l}
0 \\
1
\end{array}\right] \times\left[\begin{array}{cc}
k_{p} & k_{d}
\end{array}\right]\left[\begin{array}{c}
\Theta_{e} \\
s \Theta_{e}
\end{array}\right]}_{u_{p d}}+\underbrace{s x_{m}+\Delta_{2}}_{\Delta_{3}} \text { (8) } \\
& \text { Substituting for } X(s)=\left[\begin{array}{c}
\Theta(s) \\
s \Theta(s)
\end{array}\right], \text { Eq.8 becomes } \\
& s^{2} \Theta(s)=-\left(M_{q \dot{\alpha}}-M_{q_{n} \dot{\alpha}_{n}}+W_{2}(t)\right) s \Theta(s)-\left(W_{1}(t)\right) \Theta(s)+\Gamma \alpha_{0}\left(\frac{P_{12}}{s}+P_{22}\right) \Theta_{e}(s)+\left(k_{p}+k_{d} s\right) \Theta_{e}(s)+\Delta_{3}
\end{aligned}
$$

where $\mathrm{W}_{1}(t), \mathrm{W}_{2}(t)$ are the adaptive weights, and $\xi$ contains the failure that would eventually be corrected by the adaptive weight $\mathrm{W}_{2}(t)$.

The system open-loop transfer function is thus obtained as

$$
G(s)=\frac{\Theta}{\Theta_{e}}(s)=\frac{k_{d} s^{2}+\left(k_{p}+\Gamma \alpha_{0} P_{22}\right) s+\Gamma \alpha_{0} P_{12}}{s^{3}+\left(M_{q \dot{\alpha}}-M_{q_{n} \dot{\alpha}_{n}}+\mathrm{W}_{2}(t)\right) s^{2}+\left(\mathrm{W}_{1}(t)\right) s}
$$

If the adaptation is such that it results in a perfect model matching condition so that $\mathrm{W}_{2}(t)=M_{q \dot{\alpha}}-$ $M_{q_{n} \dot{\alpha}_{n}}$, then the open-loop transfer function in Eq. (10) becomes an ideal transfer function:

$$
G^{*}(s)=\frac{k_{d} s^{2}+\left(k_{p}+\Gamma \alpha_{0} P_{22}\right) s+\Gamma \alpha_{0} P_{12}}{s^{3}}
$$

We note that both transfer functions are functions of the $\Gamma$ and $\alpha_{0}$. The third possible open-loop transfer function is obtained when the adaptive weights reach their steady state values, assuming that the adaptation converges. Then the transfer function with constant weights is obtained as:

$$
\bar{G}(s)=\frac{k_{d} s+k_{p}}{s^{2}+\left(2 \xi \omega_{n}-2 \xi_{n} \omega_{n_{n}}+W_{2}\right) s+W_{1}}
$$

The frequency response of the three open-loop transfer functions (Eq. 10,11,12) is shown in figure 3 . The adaptive weights in the transfer functions of equations 10 and 12 are chosen after the adaptation is converged. Adaptive control system with constant adaptive gain $\Gamma=100$ is shown later to only tolerate less than 0.1 sec of time delay and have a phase margin as low as 5deg. Figure 3 indicates that the open-loop transfer function with constant weights $\bar{G}(s)$ provides a non-practical prediction for phase margin and time delay margin. Therefore $\bar{G}(s)$ should not be used for the purpose of metrics-driven adaptive control analysis. On the other hand, both the open loop transfer function $G(s)$ and the ideal transfer function $G^{*}(s)$ provide very close frequency responses, and both predict very small phase margin of about $4 \mathrm{deg}$ corresponding to $0.01 \mathrm{sec}$ time delay margin.

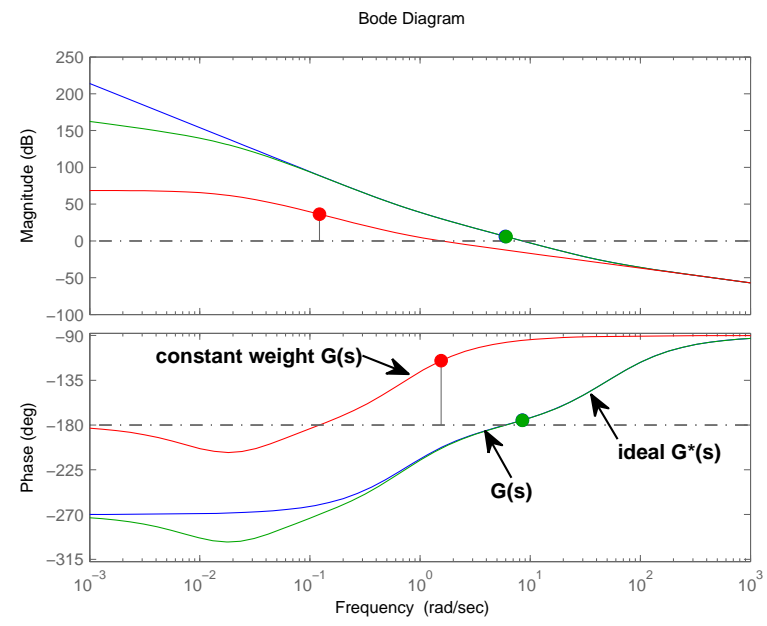

4 of 11

American Institute of Aeronautics and Astronautics 
Fig3. Frequency Response Comparison at the end of Adaptation.

Although the adaptive law provides a viable tracking performance for a system with parametric uncertainty as shown in figure 2, the system is not sufficiently robust to other types of uncertainty such as unmodeled dynamics or time delay due to the very low predicted stability margins. By adjusting the adaptive gain in order to achieve certain criteria for phase margin, adaptation is made to be metrics-driven. Since $G(s)$ contains the unknown parameter " $M_{q \dot{\alpha}}-M_{q_{n} \dot{\alpha}_{n}}+W_{2}(t)$ " due to the parametric failure, $G^{*}(s)$ is most suited for adjusting the adaptive gain and the process is explained in more details in the next section.

\section{Metrics-Driven Adaptive Control}

The ideal transfer function (Eq.11) contains all known parameters and therefore it could be used in order to adjust the adaptive gain $\Gamma$ during the adaptation in order to meet certain phase margin requirements.

The frequency response of the ideal transfer function (Eq.11) is used to define the phase margin $\phi_{m}$ and the corresponding gain corssover frequency $\omega_{g}$.

By definition, the phase margin is described as: ${ }^{5}$

$$
\phi_{m}=\arg \left[G^{*}\left(j \omega_{g}\right)\right]+\Pi
$$

or

$$
\phi_{m}=\arctan \left(\frac{\left(k_{p}+\Gamma \alpha_{0} P_{22}\right) \omega_{g}}{\Gamma \alpha_{0} P_{12}-k_{d} \omega_{g}^{2}}\right)-\pi / 2
$$

and the corresponding gain-crossover frequency $\omega_{g}$ is defined by the following expression: ${ }^{5}$

$$
\left|G^{*}\left(j \omega_{g}\right)\right|=1
$$

Or

$$
\sqrt{\frac{\left(\Gamma \alpha_{0} P_{12}-k_{d} \omega_{g}^{2}\right)^{2}+\left(k_{p}+\Gamma \alpha_{0} P_{22}\right)^{2} \omega_{g}^{2}}{\omega_{g}^{4}+\omega_{g}^{6}}}=1
$$

The $\alpha_{0}$ pareameter is computed according to Eq.7 within a given time window $T$. Using this value and a desired phase-margin $\phi_{m}$, equations 13 and 14 are solved together using a nonlinear root search to calculate $\omega_{g}$ and the appropriate adaptive gain $\Gamma$. The calculated $\Gamma$ is then used for adaptation for the next time window. With the purpose of reaching a desired phase-margin $\phi_{m}$, this process is repeated for each time window until $\Gamma$ should reach a steady state value. Therefore, the adjusted adaptive gain $\Gamma(t)$ instead of the constant gain $\Gamma$ is used in the adaptive control. The analysis result is illustrated in the simulation section.

However, analysis of the convergence of the system of nonlinear equations (13 and 14) shows that a solution for $\Gamma$ and $\omega_{g}$ always exist for the selection of the given phase margins of $\phi_{m} \leq 65 \mathrm{deg}$, and there would be no solution for $\Gamma$ or $\omega_{g}$ with the phase margins of $\phi_{m}>65 \mathrm{deg}$. This fact is shown in figure 4 , which demonstrates that only the graphs for the range of phase margins of $\phi_{m}=10-65$ deg intersect with the graph for the corresponding gain crossover frequency $\omega_{g}$ and no solution is obtained out of this range. This analysis in figure 4 is shown for $\alpha_{0}=1$, while same result is obtained for diffretent values of $\alpha_{0}$. 


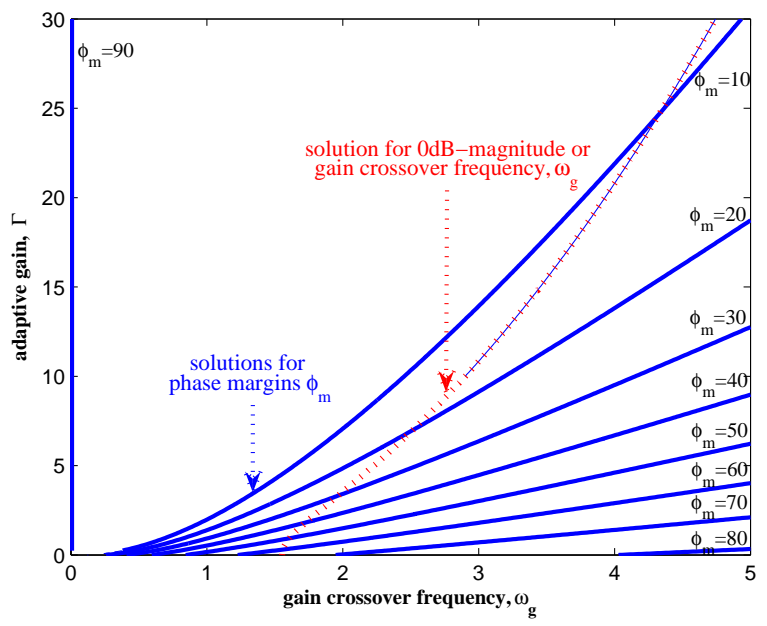

Fig. 4 Limit on the Desired $\phi_{m}$ Criteria for the Metrics-Driven Adaptive Control.

\section{Simulation}

In order to illustrate the metrics-driven adaptive control with the BLSA method a simulation is performed for a $2^{\text {nd }}$ order pitch attitude control for a generic transport model of aircraft at mach $M=0.6$ and altitude $h=20,000 \mathrm{ft}$. As mentioned in the previous section, the phase margin conforming adjusted adaptive gain $\Gamma$ is obtained from the analysis of the ideal open-loop transfer function (Eq.11) with the purpose of achieving a desired phase margin $\phi_{m}=45 \mathrm{deg}$. The adjusted adaptive gain $\Gamma$ instead of the constant gain is then used in the adaptive control. Figure 5 shows $\alpha_{0}$ that is obtained over the time-window of $T=1.5 \mathrm{sec}$, and also the adjusted adaptive gain $\Gamma$ with the starting value of $\Gamma=100$, and the steady state value of $\Gamma=1.33$.
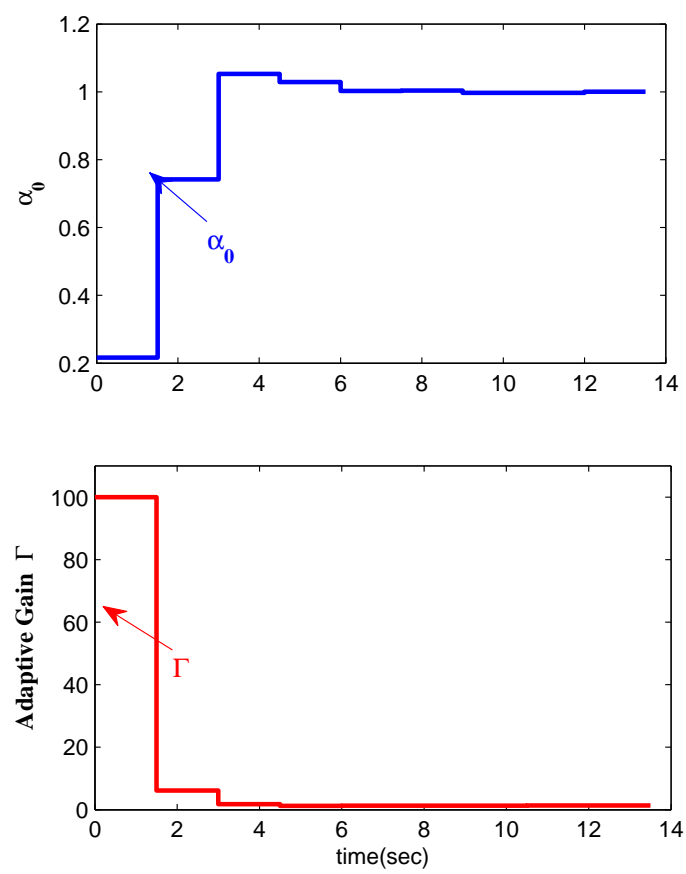

Fig5. $\alpha_{0}$ Parameter and Phase Margin Conforming Variable Adaptive Gain $\Gamma$.

Figure 6 shows the effect of the adjusted adaptive gain $\Gamma$ versus the constant adaptive gain $\Gamma=100$ on the stability margin analysis. The phase margin $\phi_{m}$ and time-delay margin $T D_{m}$ prediction by BLSA are 
calculated from the open-loop transfer function $G(s)$ (Eq.10). The calculated phase margin $\phi_{m}$ with the variable $\Gamma$ is shown to reach the desired criteria $\phi_{m}=45 \mathrm{deg}$. Also time-delay margin $T D_{m}$ is shown to be much higher with the varying adaptive gain $\Gamma$, which indicates that the adaptive system with the adjusted $\Gamma$ should be able to tolerate much more uncertainty in the system.

In order to analyze the effectiveness of the metrics-driven adaptive control, system with added uncertainty is evaluated as time delay is introduced in the simulink model right after the control law and before the plant. In figure 7 the left plot shows that the adjusted $\Gamma$ as compared to the constact $\Gamma$ does increase the time-delay margin of the system up to 15 times. The right plot shows the result of the calculation of time-delay margin by BLSA method with the adjusted and fixed $\Gamma$, and the analysis shows that BLSA provides a conservative yet practical prediction of time-delay margin with respect to the actual tolerated time-delay of the system.
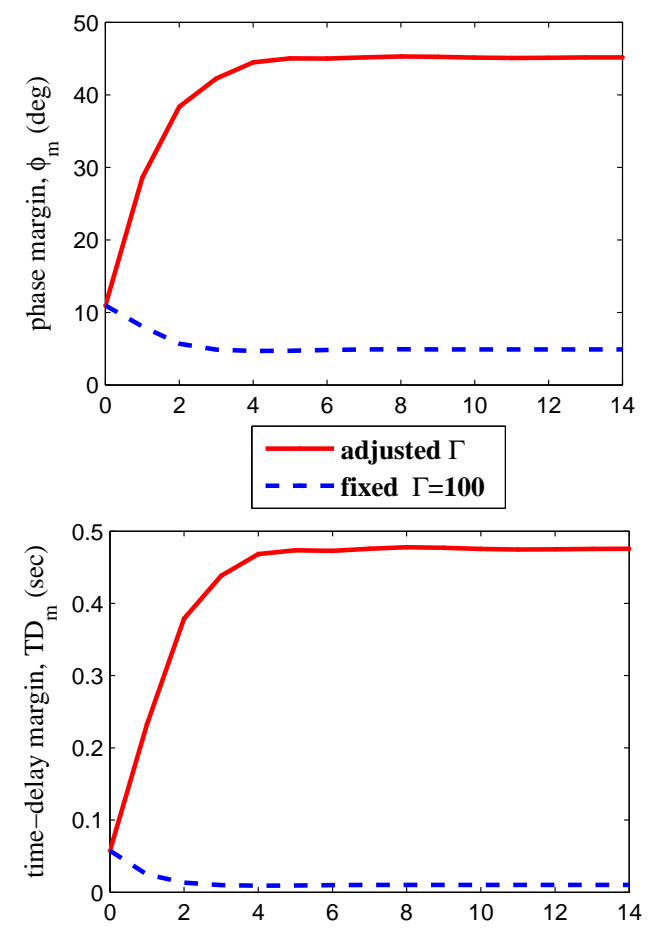

Fig 6. Stability Margin Comparison with the Adjusted and Constant Adaptive Gain $\Gamma$.
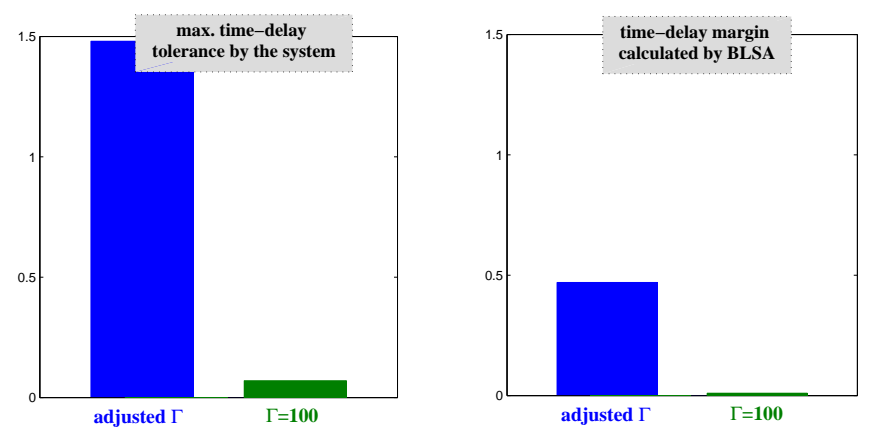

Fig 7. Analysis of Time Delay Margin Prediction with BLSA, and Max. Added Uncertainty to the System.

Simulation of the system with the adjusted adaptive gain $\Gamma$ as shown in figure 7 , indicates that system can tolerate as much as $1.48 \mathrm{sec}$ of time delay that is added after the control law. However, with the constant adaptive gain $\Gamma=100$, system can only tolerate up to $0.07 \mathrm{sec}$ of added time delay. Figure 8 illustrates the tracking performance with the introduced maximum time delay. 

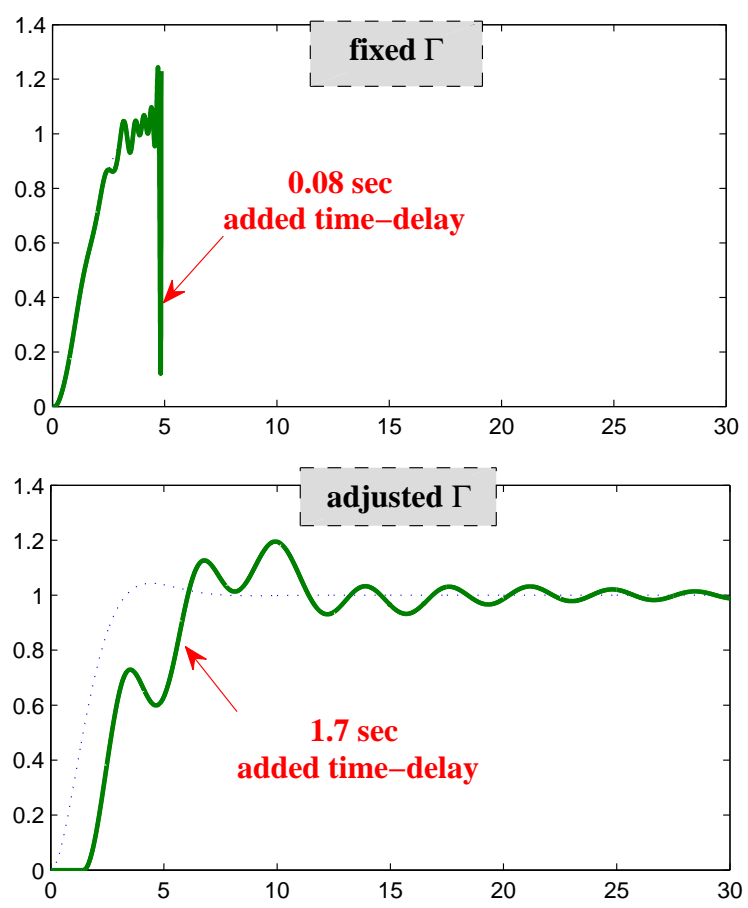

Fig 8. Tracking Performance with the Introduced Maximum Time Delay.

Performance of the adaptive weights are shown in figure 9 to demonstrate the effect of the adjusted adaptive gain $\Gamma$.

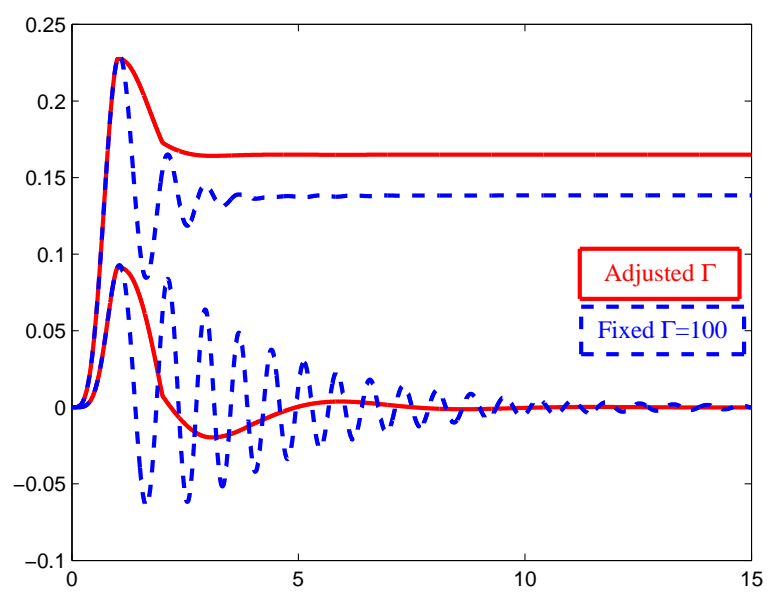

Fig 9. Performance of the Adaptive Weights with Adjusted and Fixed $\Gamma$.

Now, the choice of time-window $T$ is evaluated, over which the $\alpha_{0}$ parameter is obtained and the analysis is performed. The size of the analysis time-window $T$ has a direct affect on how frequent adaptive gain $\Gamma$ is adjusted. As illustrated in figure 10, the choice of a very small time-window like $T=0.1$ sec causes the adaptive gain $\Gamma$ to be changed very frequently. The very fast changes in the adaptive gain $\Gamma$ cause a poor convergence of the parameters since the adaptive law will not have enough time for learning. However, the analysis with a large time-window $T$ is impractical and does not capture the transient. Therefore, a trade-off should be made in choosing the proper analysis time-window $T$. 


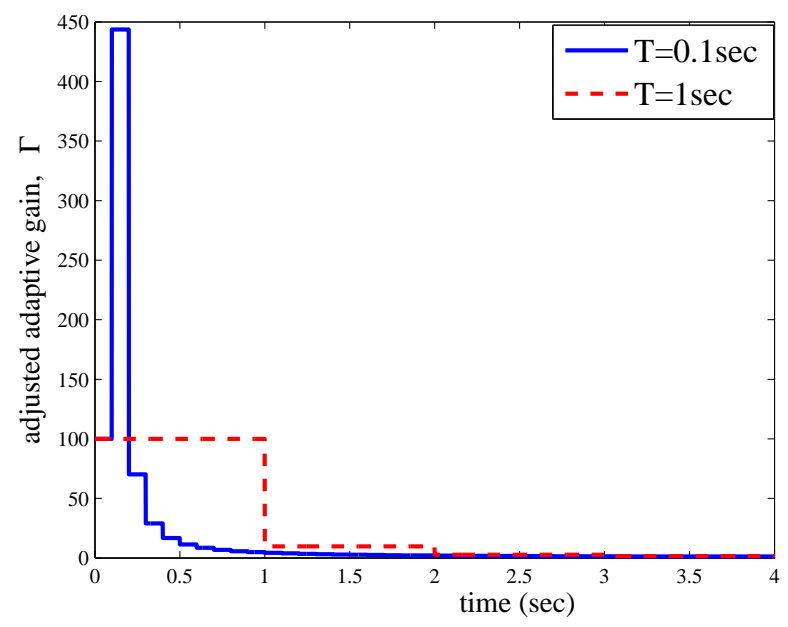

Fig10. Effect of the Analysis Time-Window $T$ on the Adjusted Adaptive Gain $\Gamma$.

As explained before, the ideal open loop transfer function (Eq. 11) that is obtained as a result of perfect parameter estimation is used to calculate the varying adaptive gain $\Gamma$ in order to acheive a certain phase margin $\phi_{m}$ criteria. The adjusted adaptive gain $\Gamma$ is used in the adaptive control, and the true off-nominal open loop transfer function (Eq. 10) is used to examine the stability margins. Based on the choice of the time-window $T$, if the adjusted adaptive gain $\Gamma$ does not cause perfect parameters convergence then the behavior of the system is affected and the desired phase margin is not reached. Figure 11 illustrates the effect of time-window $T$ on the parametric failure related index in the denominator of the true off-nominal open loop transfer function, $M_{q \dot{\alpha}}-M_{q_{n} \dot{\alpha}_{n}}+\mathrm{W}_{2}(t)$, which is supposed to reach zero after the adaptive parameters are convergenced. The analysis with different time-windows $T$ shows that the best parameter convergence is obtained with $T=1.5 \mathrm{sec}$.

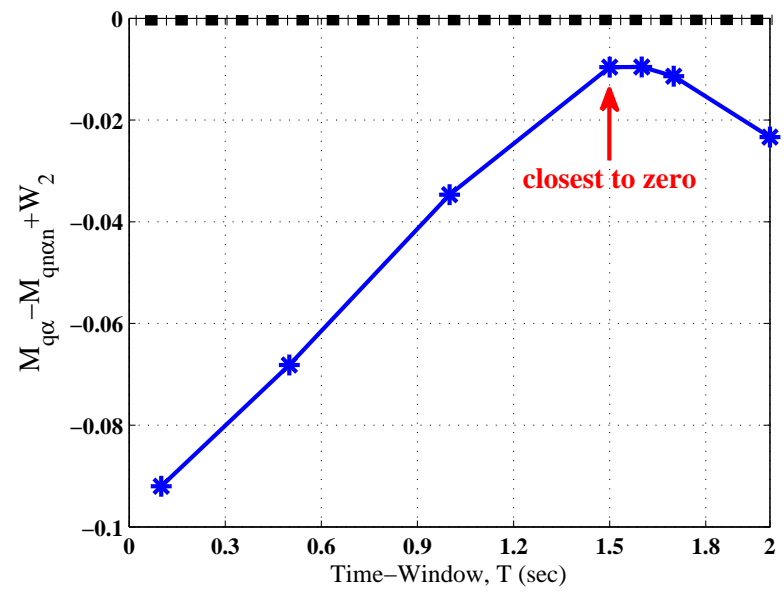

Fig11. Effect of the Analysis Time-Window $T$ on Parameter Convergence.

Figure 12 illustrates the effect of time-window $T$ on the analysis of stability margins, where the desired phase margin $\phi_{m}$ is completely achieved with $T=1.5 \mathrm{sec}$, and since the much smaller time-window of $T=0.1 \mathrm{sec}$ does not provide perfect parameter convergence, therefore the desired stability margin is not fully achieved. 


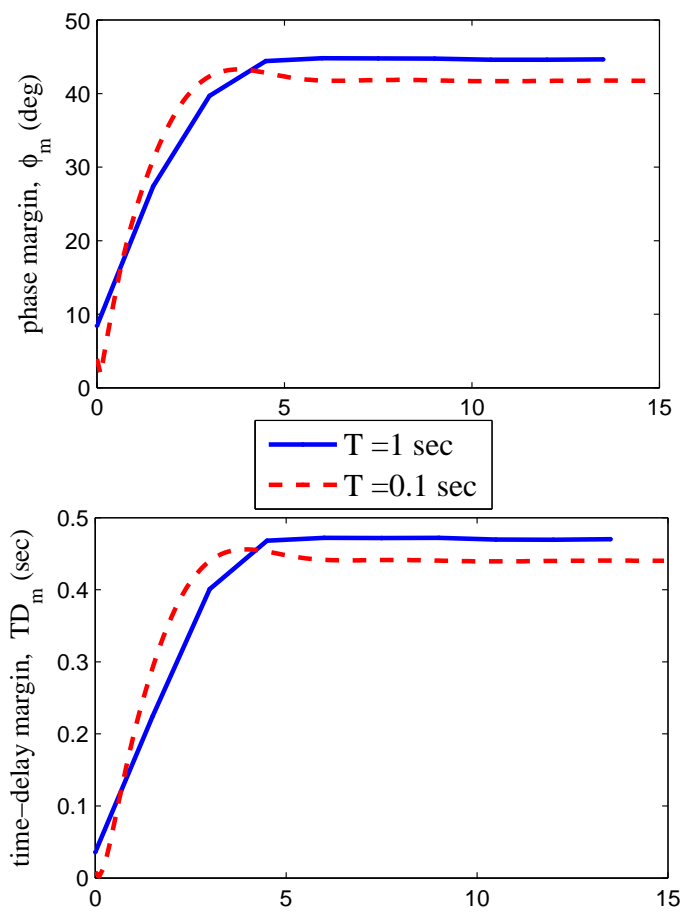

Fig12. Effect of the Analysis Time-Window $T$ on Achieving the Desired Phase Margin.

Figure 12 demonstrates a similar analysis as shown in figure 7, but with different time-windows $T=$ $0.1,1$ sec. The left plot illustrates that the max time-delay system can take is about $\% 25$ larger with $T=1 \mathrm{sec}$ as compared to $T=0.1 \mathrm{sec}$. Also, the right plot shows that the time-delay margin calculated by BLSA is almost $\% 7$ higher with $T=1$ sec.
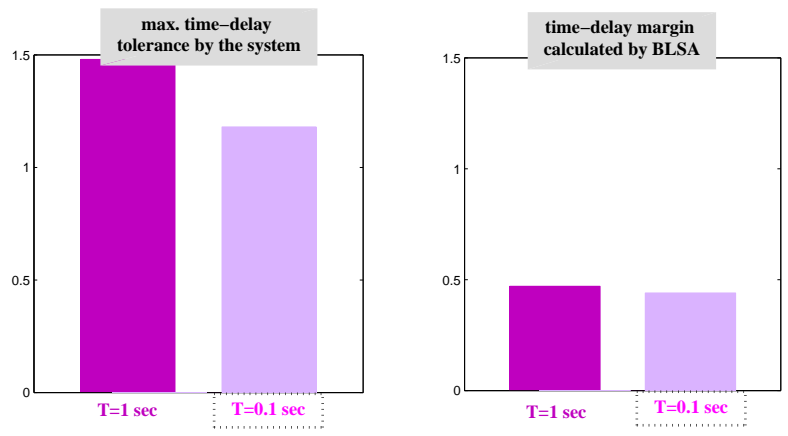

Fig.13 - Time-Delay Margin Analsys with Two Different Time-WindowsT =0.1, 1 sec.

\section{Discussion}

By using the Bounded Linear Stability Analysis (BLSA) concept, a metrics-driven learning paradigm for adaptive control system is proposed. With BLSA method a piecewise linear upper bound for the adaptive law is formed, with which the true and ideal open-loop transfer functions are formed. With the goal of achieving certain phase margin $\phi_{m}$ criteria, the ideal transfer function is used in order to adjust the adaptive gain $\Gamma$ during adaptation. As demonstrated in figure ........, the analysis indicates that the adaptive gain $\Gamma$ can always be adjusted for a specific desired phase margin on the range of $\phi_{m} \leq 65 \mathrm{deg}$.

By using the adjusted adaptive gain $\Gamma$ during adaptation, the stability margin analysis is performed for the true open loop transfer function (Eq.......... ). The analysis shows that the desired phase margin $\phi_{m}=45 \mathrm{deg}$ and a much higher time-delay margin $T D_{m}=0.48 \mathrm{sec}$ is acheived, figure......... . While by using a fixed adaptive gain $\Gamma=100$, a phase margin and time-delay margin as low as $\phi_{m}=4.9 \mathrm{deg}$ and 
$T D_{m}=0.01 \mathrm{sec}$ is obtained respectively. Furthermore, by adding a time-delay in the simulink model between the control law and plant, system with the phase margin conforming adjusted adaptive gain $\Gamma$ is shown to take as much as $1.48 \mathrm{sec}$ time-dalay and still remain stable. While system with the fixed adaptive gain $\Gamma=100$ only takes as much as $0.07 \mathrm{sec}$ time-delay. Therefore, system with the metrics-conforming variable adaptive gain $\Gamma$ becomes up to 20 times more robust to unmodeled dynamics or time delay.

The effect of the size of analysis time-window indicates that a trade-off should me made between a large time-window that does not properly capture transients and a small time-window that causes poor convergence of the adaptive parameters, figure..... .

The analysis presented in this paper

\section{Conclusion}

The application of bounded linear stability analysis on adaptive systems is evaluated in order to construct a methodology for establishing a metrics-driven learning paradigm that preserves margins during adaptation by adjusting the adaptive gain. With the purpose of achieving certain phase margin requirement, the adaptive gain is re-calculated over a time-window during adaptation. Analysis on the $2^{\text {nd }}$ order system with pitch attitude control of the generic transport aircraft proves that with the adjusted adaptive gain $\Gamma$ the system robustness to uncertainty is increased significantly, and the desired phase margin is achieved. Analysis and evaluation of the importance of selecting the proper time-window $T$ is presented. Further work will be done on elaborating on the metrics-driven learning paradigm, and also applying the method to realistic systems with different failure scenarios.

\section{References}

${ }^{1}$ J. Totah, K. Krishnakumar, and S. Vikien, "Integrated Resilient Aircraft Control - Stability, Maneuverability, and Safe Landing in the Presence of Adverse Conditions", NASA Aeronautics Research Mission Directorate Aviation Safety Program, April 13, 2007.

${ }^{2}$ N.T. Nguyen, J. D. Boskovic, "Bounded Linear Stability Margin Analysis of Nonlinear Hybrid Adaptive Control", American Control Conference, Seattle, WA, June 2008.

${ }^{3}$ R.C. Nelson, "Flight Stability and Automatic Control", $2^{\text {nd }}$ edition, McGraw-Hill Science.

${ }^{4}$ R.T. Rysdyk, and A.J. Calise, "Fault Tolerant Flight Control via Adaptive Neural Network Augmentation", AIAA Guidance, Navigation, and Control Conference, AIAA-1998-4483, 1998.

${ }^{5}$ W.K. Ho, O.P. Gan, E.B. Tay, and E.L. Ang, "Performance and Gain and Phase Margins of Well-Known PID Tunning Formulas. 